\title{
Profile Histopathology Analysis of Gastric, Duodenum, Ileum, and Colon of Inflammatory Bowel Disease (IBD) Rat Model
}

\author{
Dhita Evi Aryani *, Aulanni’am Aulanni’am, Agri Kaltaria Anisa ,Wawid Purwatiningsih \\ Faculty of Veterinary Medicine, Brawijaya University \\ *email: dhitaaryani@ub.ac.id
}

\begin{abstract}
Inflammatory bowel disease (IBD) is a disease that is mediated by the immune system that affects the gastrointestinal tract, especially the colon mucosa. This disease will cause diarrhea and mucosal damage. In general, IBD is caused by viruses and bacteria that infect the gastrointestinal tract, but some studies suggests that IBD can also be caused by side effects of non-steroidal anti-inflammatory drugs such as indomethacin. The aim of this research was to determine and compare the profile of organ histopathology, such as gastric, ileum, duodenum, and colon of normal and IBD rats. This research was a preliminary research in the course of obtaining alternative therapies for IBD derived from natural materials. Methods of this research was rats were divided into two groups, namely normal rats group and IBD rats group induced orally with indomethacin at a dose of $15 \mathrm{mg} / \mathrm{KgBB}$ once. A condition resembling IBD was present and was observed after 24 hours and the rats were euthanized. Histopathology analysis of gastric, ileum, duodenum, and colon of normal and IBD rats was done qualitatively using Hematoxylin Eosin (HE) colouring and was reported descriptively. Results of the histopathology analysis showed that some damages, such as mucosal damage, ulceration, erosion, congestion, vasodilatation, and inflammatory cell infiltration was observed in the histopathology of the gastric, duodenum, ileum and colon of IBD rats compared to the same organ histopathology of normal rats.
\end{abstract}

Keywords: inflammatory bowel disease, histopathology, indomethacin

\section{INTRODUCTION}

Inflammatory Bowel Disease (IBD) is a disease that is mediated by the immune system that affects the gastrointestinal tract, especially the colon mucosa. This disease will cause diarrhea and mucosal damage. In general causes of IBD are viruses and bacteria that infect the gastrointestinal tract, but some studies suggest that IBD may be caused due to side effects of nonsteroidalantiinflammatory drugs such as indomethacin. There are two types of IBD, namely Ulcerative Colitis (UC) and Crohn's Disease (CD)(1).

Current research on the therapeutic effect of extracts of natural products against a disease being intensively conducted. This is consistent with the slogan back to nature, so there is some cure for a disease that is combined with natural materials products. Moreover, the products of natural materials are considered to have similar therapeutic effects of modern chemical drugs with minimal side effects. And the future is expected to use this natural ingredient products can replace chemical products.

\section{OBJECTIVE}

This study was aimed to determine the profile of organ histopathology gastric, ileum, duodenum, and colon normal animals and animals IBD sufferers. 


\section{METHOD}

Methods of this research wasrats were divided into two groups, namely normal rats group and IBD rats group induced orally with indomethacin at a dose of $15 \mathrm{mg} / \mathrm{KgBB}$ once. A condition resembling IBD was present and was observed after 24 hours and the rats were euthanized (2). Histopathology analysis of gastric, ileum, duodenum, and colon of normal and IBD rats was done qualitatively using Hematoxylin Eosin (HE) colouring and was reported descriptively.

\section{RESULT AND DISCUSSION}

Histological observation of gastrointestinal tract which includes the gastric, ileum, duodenum and colon of rats result indomethacin induced inflammatory bowel disease models include the condition of the mucosal lining, erosion, congestion, rupture villi, inflammatory cell infiltration, and vasodilatation.

\subsection{Histopathology of Gastric}

Histopathological profile Rattus norvegicus gastric is shown in Figure 1, where the image A is histopathology of gastric normal (negative control) and the B is a picture of IBD's gastric histopathology. Microscopic picture negative control showed normal shape hull seen their layers of clear, namely the mucosal layer (M), muscularis mucosa (MM), and submucosal (SM). One of the characteristics of a microscopic picture is the gastric pit (GP) which can be elongated up to half the depth of the mucosal layer. The mucosal layer appears the pyloric glands / pyloric gland (PG). While in the submucosal layer contained blood vessels-arteries / blood vessels (BV) is large (Figure 1A). In rats with Indomethacin induction show damage to the mucosal layer, ulcer, congestion on mucosal lining,loss of surface cells and the structure of gastric mucosal pit and blood vessel vasodilatation(Figure 1B).
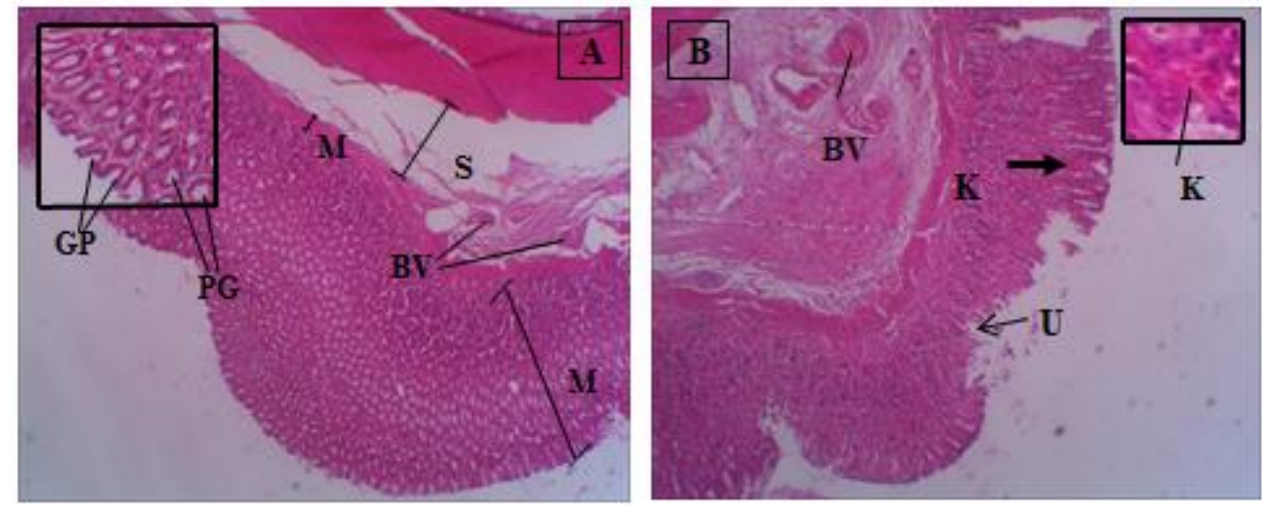

FIGURE 1. Microscopic picture of the gastric of mice (magnification 40x)

Description: (A) normal gastric; (B) IBD's gastrics; (U) ulcers; (K) congestion; (MM) muscularis mucosa; (SM) submucosal; (M) mucosa; (PG) pyloric gland; (GP) gastric pit

\subsection{Histopathology of Duodenum}

Histopathological profile Rattus norvegicus duodenum is shown in Figure 2 where the image $\mathrm{A}$ is the image of histopathology duodenum normal (negative control) and the $\mathrm{B}$ picture is a picture of histopathology duodenum IBD's.Microscopic picture duodenum normal mice showed normal form duodenum, the layer muscularisexterna (ME), submucosal $(\mathrm{SM})$, and mucosal (M) are clear. Brunner's gland (BG) in the submucosal layer and villi (V) 
which is shaped like a leaf in the mucosal layer looks normal. No damage appears in the duodenum (Figure 2A).In IBD's mice duodenumshown duodenum damages, include rupture of the villi, congestion on external mucous layer,inflammation characterized by infiltration of inflammatory cells and macrophages(Figure 2B).
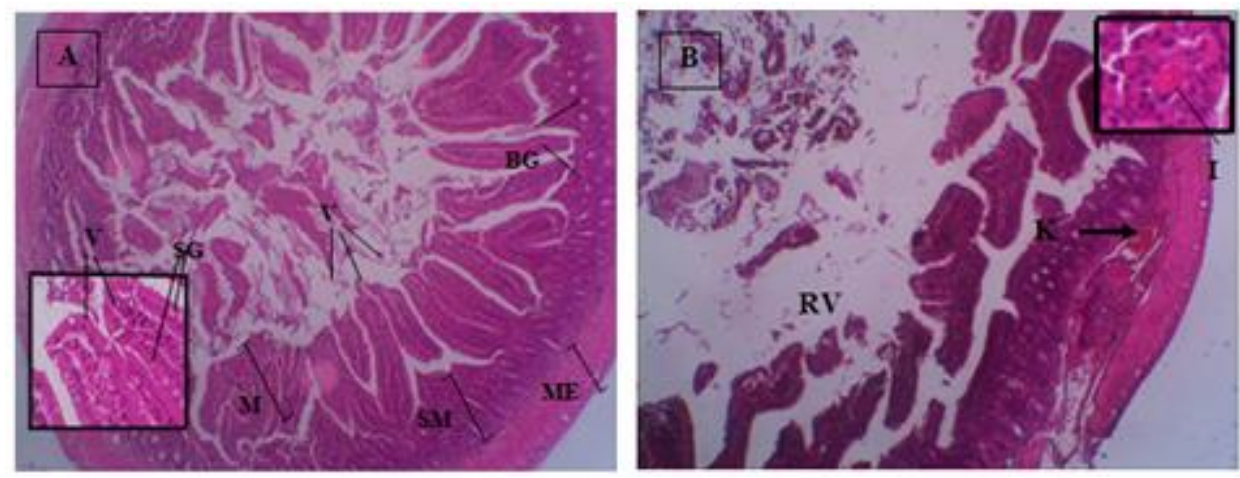

FIGURE 2. Microscopic picture Rattusnorvegicusduodenum (magnification 40x)

Description: (A) normal duodenum; (B) induction of duodenal results indomethacin $15 \mathrm{mg} / \mathrm{kg}$; (ER) erosion; (K) congestion; (RV) rupture villi; (I) Inflammation; (ME) external muscle; (SM) sub mucosa; (M) mucosa; (V) villi; (SG) goblet cells; (BG) brunner gland

\subsection{Histopathology of ileum}

Histopathological profile Rattus norvegicus ileumis shown in Figure 3 where the image A is the image of histopathology ileum normal (negative control) and the B picture is a picture of histopathologyi ileumIBD's. The negative control showed a apparent lack disintegritas on mucosal surfaces and do not experience erosion, appeared villi ileum shaped like a mace, visible goblet cells was striking on the surface villi, appears kriptaliberkuhn and muscularis mucosa (Figure 3A).On IBD's ileum obtained with erosion,disintegritas mucosal surface and inflammatory cell infiltration (Figure 3B).
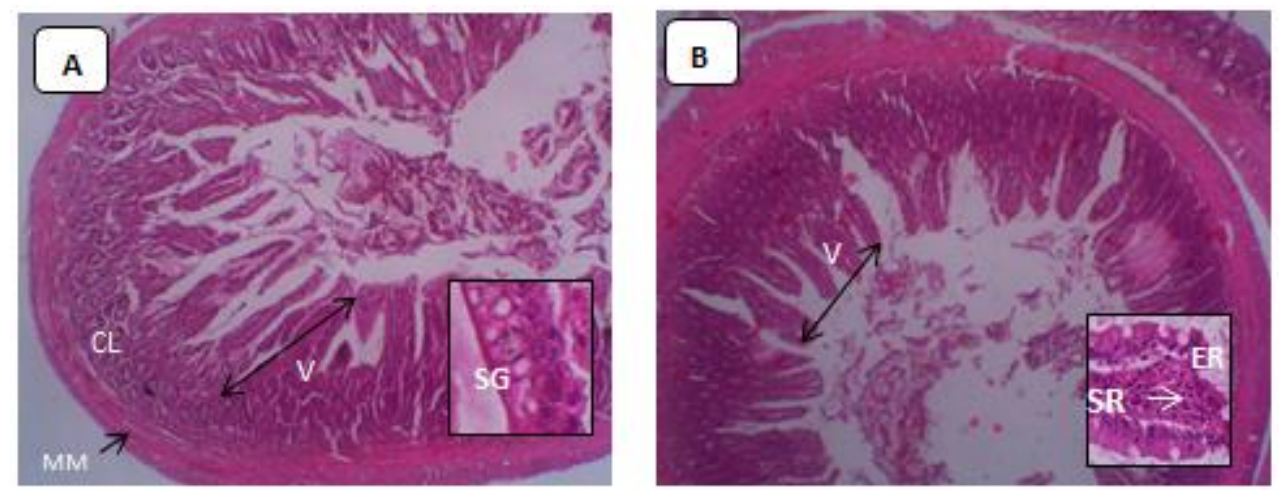

FIGURE 3. Histopathologic features ileum with 40x magnification. A. ileum normal (negative control). B. ileum results of induction of indomethacin $15 \mathrm{mg} / \mathrm{kg}$ (positive control). Description: KL: crypt Liberkuhn, V: villi, SG: goblet cells, MM: muscularis mucosa, SR: inflammatory cells, ER: erosion

\subsection{Overview Histopathology Colon}

Histopathological profile Rattus norvegicus colon is shown in Figure 4 where the image A is the image of histopathology normal colon (negative control) and the B picture is a picture of histopathologyIBD's colon. Microscopic observation on the negative control showed the mucosal surface is not eroded and does not seem any disintegrates lining of the colon, which are straight and muscular external circular and longitudinal thick (Figure 4A). 
And on IBD's colon was noted that indicates congestion in the mucosal layer, muscularis external circular and longitudinal looks thinner than the negative control (Figure 4B).
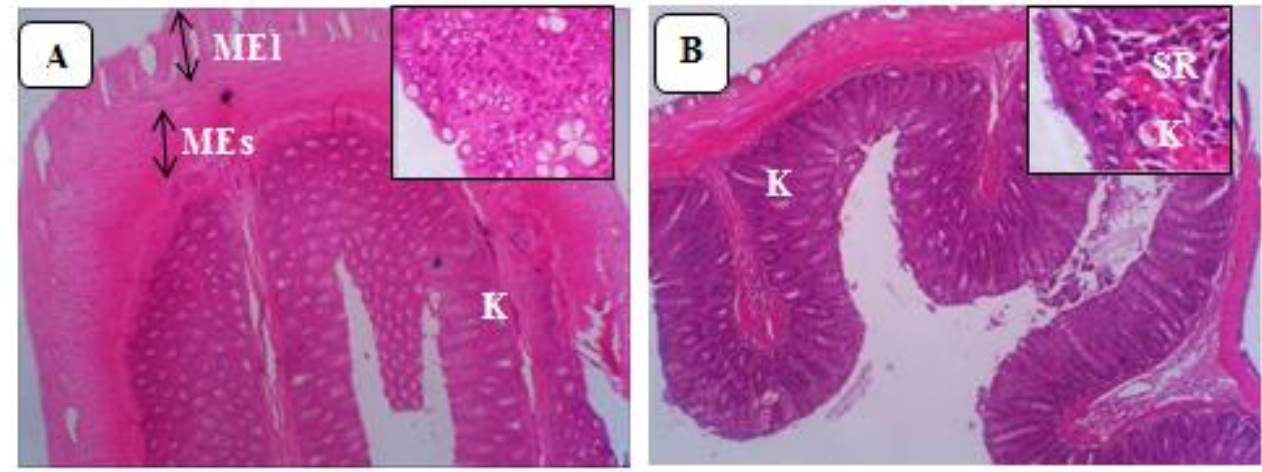

FIGURE 4. Histopathologic features of the colon with 40x magnification. A. normal colon (negative control) Description: Mel: Longitudinal External mucosa, Mes: External Circular mucosa, KL: crypt Liberkuhn, K: Congestion, SR: Inflammatory Cells

This study using indomethacin as the induction of inflammatory bowel disease as indomethacin could blockade the COX-1 pathway resulting in decreased prostaglandin synthesis and the amount of mucus in the gastrointestinal tract (3). Decrease the amount of mucus will cause disruption of normal gastrointestinal microflora and the loss of the mucosal barrier, thus facilitating the occurrence of inflammation. In addition indomethacin can trigger the production of Reactive Oxygen Species (ROS), which in turn can trigger an inflammatory reaction, aggregation and activation of neutrophils and the release of a protease enzyme. This has the advantage of the use of indomethacin can induce damage in the ileum and jejunum, but theinflamation not localized in the colon. Therefore, the results of this study in part of the colon is not a lot of damage $(4,5 \& 6)$.

\section{CONCLUSION}

The conclusion of this study is there were differences between the profile of organ histopathology gastric, ileum, duodenum, and colon normal animals and animals IBD sufferers. And Indomethacin can be used as inducer Inflamatory Bowel Disease on Rattus norvegicus.

\section{ACKNOWLEGMENT}

This project supported by Funding from Faculty of Veterinary Medicine Brawijaya University through DPP/SPP 2016 and facilitated by Veterinary Faculty of Brawijaya University's Clinic Department Team and Biochemistry Department Team.

\section{REFERENCES}

1. Friedman, Sonia and Richard S.B. 2010. Inflamatory Bowel Disease. In : Longo, DL. And AS. Fauci. Harrison's Gastroenterology and Hepatology. The McGraw-Hill Company. 2010 pp.

2. Aulanni'am; A.Roosdiana and NL. Rahmah,The Potency of SargassumduplicatumBory Extract on Inflamatory Bowel Disease Theraphy in Ratusnorvegicus, Journal of Life Sciences, 2012, vol 6 pp. 144-154

3. Lacy CF, Armstrong LL, Goldman MP and Lance LL,Drug Information Handbook, $18^{\text {th }}$ Ed., American Pharmacist Association, USA : Lexi Comp, Inc. 2010 
4. Yamada T, Deitch E, Specian RD, Perry MA, Sartor RB, Grisham MB. Mechanisms of Acute and Chronic Inflammation Induced by Indomethacin. Inflammation, 1993,vol 17 pp. 641-662.

5. Piepoli AL, De Salvatore G, De Salvia MA, Mitolo CI, Siro-Brigiani G, Marzullo A, Grattagliano I, Mitolo-Chieppa D, Palasciano G, Portincasa P.,Indomethacin-induced ileitis is associated with tensiometric, vascular and oxidative changes in the experimental rat model. EurJ Clin Invest, 2005, vol. 35 pp. 271-278.

6. Szalai, Zit, KrisztinaKupai, Medea Veszelka, AnikoPosa, SzilviaTorok, Aniko MB., ZoltanBarath, Ferenc AL and CsabaVarga,Novel Features of the Rat Model of Inflammatory Bowel Disease based on 2,4,6-trinitrobenzenesulfonic acid induced Acute Colitis. ActaBiologicaSzegediensis, 2014,Vol. 58(2) pp. 127-132 